\title{
Geological Implications of a Physical Libration on Enceladus
}

\author{
T.A. Hurford ${ }^{a, *}$, B.G. Bills ${ }^{\text {b,c }}$, P. Helfenstein ${ }^{\mathrm{d}}$, R. Greenberg ${ }^{\mathrm{e}}$, \\ G.V. Hoppa ${ }^{\text {f }}$, D.P. Hamilton ${ }^{\mathrm{g}}$ \\ a Planetary Systems Laboratory, NASA Goddard Spaceflight Center, Greenbelt, MD \\ 20771, USA. \\ b Planetary Geodynamics Laboratory, NASA Goddard Spaceflight Center, \\ Greenbelt, MD 20771, USA. \\ ${ }^{\mathrm{c}}$ Institute for Geophysics and Planetary Physics, Scripps Institution of \\ Oceanography, La Jolla, CA 92093, USA. \\ ${ }^{\mathrm{d}}$ CRSR, Cornell University, Ithaca, NY 14853, USA. \\ ${ }^{\mathrm{e}}$ Lunar and Planetary Laboratory, University of Arizona, Tucson, AZ 85721, \\ USA. \\ ${ }^{\mathrm{f}}$ Raytheon, Woburn, MA 01801, USA. \\ ${ }^{g}$ Department of Astronomy, University of Maryland, College Park, MD 20742, \\ $U S A$.
}

\begin{abstract}
Given the non-spherical shape of Enceladus (Thomas et al., 2007), the satellite will experience gravitational torques that will cause it to physically librate as it orbits Saturn. Physical libration would produce a diurnal oscillation in the longitude of Enceladus' tidal bulge which, could have a profound effect on the diurnal stresses experienced by the surface of the satellite. Although Cassini ISS has placed an
\end{abstract}


observational upper limit on Enceladus' libration amplitude of $F<1.5^{\circ}$ (Porco et al., 2006), smaller amplitudes can still have geologically significant consequences. Here we present the first detailed description of how physical libration affects tidal stresses and how those stresses then might affect geological processes including crack formation and propagation, south polar eruption activity, and tidal heating. Our goal is to provide a framework for testing the hypothesis that geologic features on Enceladus are produced by tidal stresses from diurnal physical and optical librations of the satellite.

Key words: Tidal Stress, Libration, Tectonics, Enceladus

\section{Introduction}

The Cassini spacecraft observed plumes actively erupting from the south polar region of Enceladus (Porco et al., 2006), and the heat emanating from the south polar region has been estimated to be $\sim 6 \mathrm{GW}$ (Spencer et al., 2006). This came as quite a surprise since this small moon of Saturn (Radius = $250 \mathrm{~km}$ ) was expected to have cooled long ago, based on estimates of heating within the satellite. These estimates place current radiogenic heating at 0.32 GW (Schubert et al., 1986; Porco et al., 2006) and tidal heating from its small orbital eccentricity at just $0.12 \mathrm{GW}$ if $k_{2}$ is 0.0018 and $Q$ is 20 (Yoder, 1979; Porco et al., 2006). Together these two sources of heating provide only about 0.4 GW, less than a tenth of the observed heat emanating from the south pole.

However, the estimates of tidal heating rely on the tidal response of Enceladus that may be too conservative, so tidal heating may be greater than expected.

* terry.a.hurford@nasa.gov (Terry A. Hurford) 
In fact, recent work by Hurford et al. (2007) and Nimmo et al. (2007) require a larger tidal deformation of Enceladus in order to produce sufficient tidal stress to open cracks on the surface and generate heat through tidal shearing. This larger tidal response implies $k_{2}$ must be at least 0.12 , implying at least 8 GW of tidal heating. Even though this amount of heating might be sufficient to explain the $\sim 6 \mathrm{GW}$ observed at the south pole, it is a global budget and therefore it is unlikely that most of this tidal heating would be focused in a region near the south pole.

Even more heat could be dissipated by a physical libration of Enceladus as it orbits Saturn (Wisdom, 2004). Fits to Enceladus' physical shape from Voyager data indicated that the direction of the Saturn-pointing location on Enceladus may undergo a small oscillatory motion with a natural frequency of $1 / 3$ of the orbital frequency (Wisdom, 2004), producing greater tidal heating. New fits of Enceladus shape using Cassini data showed that Enceladus was closer to the 4:1 secondary resonance and not the 3:1 secondary resonance (Porco et al., 2006). Cassini placed an upper limit on the amplitude of this libration of $1.5^{\circ}$.

Although the upper limit on the libration amplitude for Enceladus seems small, this spin state has significant ramifications for the satellite. The physical libration affects the tidal stress and thus will influence all processes in which stress plays a dominant role. Although Enceladus' physical libration may not be directly detectable by Cassini, the effect of the physical libration may be observable, providing indirect evidence of Enceladus' libration state. 


\section{Tidal Stress on a Librating Body}

To simplify calculations, we make a thin shell approximation that yields the surface stresses from the tidal deformation of an elastic outer layer, assuming that this layer is decoupled from the deeper interior of Enceladus (e.g. by a fluid layer or a solid layer that behaves as a fluid on the time scale of the stress). That is to say there is negligible shear between the elastic shell and the interior. The thin elastic layer cannot affect the tidal distortion and thus deforms to fit the tidal figure taken of Enceladus, stretching and producing stress on its surface. The stresses on the surface are given by the VeningMeinesz equations:

$$
\sigma_{\theta \theta}=-\frac{3 M h_{2} \mu}{8 \pi \rho_{a v} a^{3}}\left(\frac{1+\nu}{5+\nu}\right)(5+3 \cos 2 \theta)
$$

and

$$
\sigma_{\phi \phi}=\frac{3 M h_{2} \mu}{8 \pi \rho_{a v} a^{3}}\left(\frac{1+\nu}{5+\nu}\right)(1-9 \cos 2 \theta)
$$

where $\theta$ is a surface point's angular distance from the axis of deformation symmetry (Melosh, 1977; Leith and McKinnon, 1996; Greenberg et al., 1998). In these expressions $M$ is the mass of the tide-raiser, Saturn, $\mu$ is the rigidity while $\nu$ is the Poisson's ratio of the thin elastic shell, $h_{2}$ is the Love number describing the response of the body to the tide raising potential, $\rho_{a v}$ is the average density of Enceladus and $a$ is the semi-major axis of its orbit, which describes the average distance to Saturn. $\sigma_{\theta \theta}$ is the stress along the surface in the direction radial to the axis of symmetry, while $\sigma_{\phi \phi}$ is the stress along the surface in a direction orthogonal to the $\sigma_{\theta \theta}$ stress. In the convention here positive stresses are compressional and negative stresses tensile. 
Note that $\sigma_{\theta \theta}$ is always tensile, while $\sigma_{\phi \phi}$ is tensile for $\theta<42^{\circ}$ (near the tidal symmetry axis) and compressional for larger $\theta$. Furthermore note that $\sigma_{\theta \theta}=\sigma_{\phi \phi}$ at $\theta=0^{\circ}$ as required by symmetry. The direction of these stresses can be understood from considering tidal deformation which elongates a spherical body to form two bulges at $\theta=0^{\circ}$ and $\theta=180^{\circ}$ while shrinking the circumference of the great circle at $\theta=90^{\circ}$.

Enceladus' finite orbital eccentricity causes small daily changes in the distance between Enceladus and Saturn, affecting the height of the tide. During an orbit, the height of the main tide oscillates with an amplitude $\left(9 e h_{2} M R\right) /\left(4 \pi \rho_{a v} a^{3}\right)$ where $e$ is the orbital eccentricity and $R$ is Enceladus' radius. With our assumption that $h_{2}$ is 0.32 the diurnal tidal amplitude would be $\sim 5 \mathrm{~m}$.

Besides affecting the height of the tide on Enceladus, the orbital eccentricity also causes the longitude of the tidal bulge to oscillate as it tracks the position of Saturn throughout an orbit. This oscillation is called the "optical libration" in longitude. The term was coined by Isaac Newton to describe the apparent change in longitude of features on the Moon as described by Galileo in 1637 (Newton, 1686).

Even if the eccentricity were zero, a moon can experience a "physical libration" or an actual wobble of the moon about its own center of gravity. This physical libration would also result in the oscillation of the tidal bulge in longitude with respect to a fixed location. The physical libration is composed of a both a free and forced component.

The free libration period of Enceladus depends on the shape of the satellite and the distribution of mass within its interior. Tidal dissipation should have damped this libration to zero amplitude. However if the free libration period 
is commensurate with the orbital period, a spin-orbit resonance occurs which may increase the amplitude of this libration. Based on Cassini ISS data of Enceladus' shape the free libration period estimated to be about 4 times the orbital period (Porco et al., 2006), possibly implying Enceladus might be in such a spin-orbit resonance. However, for this paper we assume that the free libation has been damped to zero amplitude.

The other component of the physical libration, the forced libration, is driven by torques from Saturn on the surface of the satellite. Enceladus' forced libration responds at the frequency of the forcing, which would likely be the satellite's orbital mean motion $n$. The amplitude of the forced libation depends on the interior properties of the satellite. Moreover, if the icy crust of Enceladus is decoupled from the deeper interior by a liquid layer, a larger forced libration amplitude may be possible. To the extent that tidal heating influences the mean thickness and lateral variation in local thickness of the ice shell, the amplitude and phase of the forced libration may likely change over time.

The orbital eccentricity (optical libration) and rotational (physical libration) effects combine, yielding the diurnal, or daily, oscillation of the tide, as illustrated schematically in Fig. 1. The longitude of the tide, relative to a fixed longitude, at any point throughout the orbit is the difference between the optical and physical libration or

$$
-2 e \sin (n t)+F \sin (n t+\psi)
$$

where $2 e$ is the amplitude of the optical libration, $n$ is the mean motion, and $t$ the time since pericenter passage and $F$ is the amplitude of the physical libration, which has a phase $\psi$ relative to the optical libration. $\psi=0^{\circ}$ would 
be a correspond to a physical libration that is "in-phase" with the optical libration, while $\psi=180^{\circ}$ would be "out-of-phase". The oscillatory motion of the tidal bulge produces daily changes in $\theta$ in Eq. 1 and Eq. 2. The changes in $\theta$ plus daily changes in the height of the tide combine, yielding the diurnally varying part of the tide, producing elastic stress on the surface (See Appendix A).

The three cases illustrated in Fig. 1, have identical tidal stress fields at pericenter and also at apocenter. (c.f. Greenberg et al. (1998); Greenberg (2005)). However, the three stress fields differ when the satellite is at $n t=\pi / 2$ as can be seen in Fig. 2. The stresses associated with $1.5^{\circ}$ out-of-phase physical librations (Figs. 2b) is enhanced in magnitude relative to case (a) with no physical libration. Although the magnitude of the tidal bulge is the same in both cases, the longitude of the tidal bulge moves further from zero, producing more stress in case (b). For in-phase physical librations (case c), the tidal stress field (Fig. 2c) is enhanced in magnitude and the principle stresses are rotated by $90^{\circ}$. Here the longitude of the tidal bulge is on the opposite side of the $0^{\circ}$ meridian, reversing the directions of tension and compression.

Although Fig. 2 shows the tidal stress field at one location in the orbit, the tidal stress field is constantly changing. In the case in which the satellite exhibits no physical libration, zones of tension and compression at the equator move eastward throughout the orbit. Moreover, the orientation of the principle stresses rotate in a counterclockwise fashion in the northern hemisphere and clockwise in the southern hemisphere. When the amplitude of the physical libration is small compared to the optical libration $(F<c 2 e)$ or the physical libration is out of phase with the optical libration, this pattern of how the stress changes throughout the orbit remains the same, even if the magnitude 
of the stresses are affected (the white region in Fig. 3). However, when the amplitude of the physical libration is equal to or greater than the amplitude of the optical libration and relatively in phase with the optical libration (the black region in Fig. 3), the zones of tension and compression at the equator move westward throughout the orbit and the orientation of the principle stresses rotate in a clockwise fashion in the northern hemisphere; counterclockwise in the southern hemisphere. In a special case, where the amplitude of the physical libration is exactly equal to the amplitude of the optical libration $(F=2 e)$ and in phase with it $(\psi=0)$, the orientation of the principle stresses do not rotate throughout the orbit and the zones of tension and compression remain stationary, while oscillating between compression and tension. Along the gray

line in Fig. 3, defined by $\cos \psi=2 e / F$, the tidal stress field changes its sense of rotation at pericenter and apocenter and the zones of tension and compression oscillate about a fixed longitude. It follows from Eq. 3 that the oscillation of the tidal bulge would be at its maximum eastward or westward position at pericenter and apocenter if $\cos \psi=2 e / F$.

\section{Effects on Tidal Processes}

Because a physical libration affects the diurnal stress field as shown above, it may influence the processes in which tidal stress play a dominant role.

\subsection{Tidal Control of Eruptions}

In addition to the plumes that were detected near the south polar region of Enceladus large rifts in the crust were discovered near the south pole, which 
are evidently the source of the plumes (Porco et al., 2006; Spencer et al., 2006; Spitale and Porco, 2007). These features informally called "tiger stripes", exhibit higher temperatures than the surrounding terrain and are likely sources of the observed eruptions (Spencer et al., 2006). By a method developed to consider stress along cracks on Europa (Greenberg et al., 1998; Hoppa et al., 1999a), diurnal variation in the surface stress normal to a fault can be calculated. Over Enceladus' orbital period, stresses across the tiger stripes alternate from compressive to tensile, allowing the faults to open, which could expose a subsurface volatile reservoir, creating an eruption (Hurford et al., 2007). The state of the stress across the fault depends on the azimuthal orientation (the "strike") of the fault as well as on its location.

A physical libration changes the stresses on the tiger stripes, especially the timing of the transition between tension and compression, which could affect the timing and location of eruptions. Fig. 4a shows the stresses on the tiger stripes during $\pi / 4<n t<\pi / 2$ for the case with no physical libration (corresponding to Fig. 1a). During this period large portions of the tiger stripes are transitioning from compression to tension (shown by yellow in Fig. 4a). However, if Enceladus exhibits a physical libration the timing of this transition changes. For example in the case where the physical libration has an amplitude of $F=1.5^{\circ}$ that is out of phase with the optical libration ( Fig. 4b), during the same eighth of an orbit period most of the tiger stripes remain in tension, having transitioned from compression to tension during the period $0<n t<\pi / 4$. Finally, for the same physical libration amplitude, but in phase with the optical libration (Fig. 4c), the stresses on the tiger stripes during this part of the orbit are mostly compressive.

During each orbit, every portion of each tiger stripe rift spends roughly half 
the time in tension, which may expose volatiles and allow eruptions. Then, within a few hours, once again the stress becomes compressive, forcing cracks to close, ending any possibility of an eruption until the next cycle. Therefore, eruption rates may vary periodically, affecting the injection of material into Saturn's E ring.

In a simple model, eruption rates may be proportional to the total length of tiger stripe segments in tension at any moment. Fig. 5 shows the fraction of the tiger stripes in tension, our proxy for eruption rate, for the three cases of Fig. 1. In the case of no libration, eruptions rates might be high during the first half of the orbit and low during the second half. An out-of-phase physical libration enhances this pattern, since the tiger stripes open more quickly after pericenter and close just after apocenter passage. The opposite is seen for a physical libration that is in phase with the optical libration. Certain distributions of eruption activity, therefore, would be indicative of physical libration.

\subsection{Tidal Shear Heating}

While periodic stress across the tiger stripes may control eruption events, in a complementary process, periodic shear stress along the rifts may generate heat along their lengths (Nimmo and Gaidos, 2002; Prockter et al., 2005; Nimmo et al., 2007). The shear stress can drive strike-slip motion along the fault and in turn can dissipate energy through frictional heating. According to Nimmo et al. (2007), this additional source of heat may have the capacity to enhance eruptions, although that model depends on the assumed rate of strike-slip displacement. 
In that model, heat generated by tidal shear stress along rifts in the south polar region of Enceladus have been compared with the locations of hotspots observed by Cassini's Composite Infrared Spectrometer (CIRS) (Spencer et al., 2006). There is a reasonable correlation between hotspot locations and predictions of tidal heat generation along the Damascus fault. However, on the Baghdad fault, CIRS detected the hottest region near the south pole, which does not fit the tidal shear heating model (Spencer et al., 2006; Nimmo et al., 2007).

The mismatch between the theory of tidal shear heating and the observations of heat on Enceladus may be due to over simplifications in the model of tidal shear heating by Nimmo et al. (2007). One possibility important effect that was neglected are physical librations which affect the diurnal tidal stress and therefore the amount of tidal shear heating along rifts.

Fig. 6 illustrates how a physical libration can affect tidal shearing along the tiger stripes. In Fig. 6a the amount of shear experienced along the tiger stripes is calculated in a similar method as was described by Nimmo et al. (2007), except we use a thin shell approximation for the diurnal tidal stress on Enceladus' surface. The results shown in Fig. 6a reproduce earlier results by Nimmo et al. (2007). However, when a physical libration is added the pattern of tidal shear, and hence the amount of heat generated and the locations of hot spots are also affected. In the case of the libration shown in Fig. 6b (physical libration of $1.5^{\circ}$ in amplitude with a phase of $270^{\circ}$ ), areas of high shearing seen in Fig. 6a are still present, but new areas of high shearing have emerged. In particular, our result with libration includes extra shear near the south pole, a better match to the heat measured there by CIRS. This agreement provides indirect evidence (albeit model-dependent) that Enceladus may undergo phys- 
ical libration.

\subsection{Strike-slip Motion}

In a method similar to that used to consider stress along cracks on Europa's surface, diurnal variation in the surface stress on a fault can be calculated (Hoppa et al., 1999a). Tidal stress along a fault can produce strike-slip displacement by a process analogous to walking (Hoppa et al., 1999a). In tidal walking faults open and close out of phase with left- and right-lateral shear producing small, net, daily offsets along the faults. When the stress normal to a fault is tensile, the fault opens allowing shear stress to produce an offset. About half an orbit later, the stress normal to the fault changes from tension to compression, closing the fault. Once closed, friction along the fault limits the ability of shear stress to completely relieve the entire offset. After one cycle, the fault displays a small net sense of strike-slip displacement along its length. Over many successive cycles of opening and closing the small strike-slip offsets build, producing a greater amount of strike-slip displacement. Such displacements may ultimatley be observed on Enceladus too with Cassini Imaging Science Subsystem (ISS) images.

The sense (left- or right-lateral) of strike-slip displacement along a fault depends predictably on the orientation and location of the fault, according to the tidal walking theory (Hoppa et al., 1999a). Without physical libration, strike-slip displacements poleward of $60^{\circ} \mathrm{S}$ should all be right-lateral in sense (Hoppa et al., 1999a) because stresses in this region rotate in a clockwise direction throughout Enceladus orbit. 
However, when we add the effect of physical libration, tidal walking can yield left-lateral strike-slip displacement in the south polar region (Fig. 7), if the amplitude of the physical libration is greater than the optical libration $(2 e$ radians, i.e. $F>0.54^{\circ}$ ) and the libration is relatively in phase. In such a case, the stress field throughout the orbit rotates in a counter-clockwise direction, allowing left-lateral strike-slip displacement.

If the relative phase between the physical and optical libration changes with time, right-lateral offsets would form along strike-slip faults when the two librations are relatively out of phase with each other, but left-lateral offsets would form when the two librations are in phase with each other (Fig. 7). If the amplitude of the physical libration were less than amplitude of the optical libration $(F<2 e)$, only right lateral strike-slip offsets should be produced, regardless of the libration phase.

\subsection{Initial Formation of Cracks}

One prominent tiger stripe near Enceladus' south pole consists of arcuate segments, resembling the shape of cycloidal cracks on Europa (c.f. Hurford et al. (2007)). On Europa, these distinctive tectonics patterns were likely produced by periodic tidal stresses (Hoppa et al., 1999b). As the cracks form and propagate across the surface, their paths are affected as the stresses rotate in the region. The similarity between Enceladus' tiger stripes and Europa's cycloidal ridges is in shape only; they are quite distinct from one another morphologically. Nevertheless, the similar shape of the tiger stripe suggests that its formation may have been similarly controlled by diurnal tidal stresses (Hurford et al., 2006, 2007). Subsequent modification of the surface along the 
tiger stripes on Enceladus likely differed from the ridge-forming processes on Europa.

On Enceladus with just stress from the optical libration, the arcuate rift would not have formed at its current location (Hurford et al., 2006, 2007). The variations in the stress field at this longitude cannot recreate the shape of the feature. However, as with Europa (Hoppa et al., 2001), the formation of the feature may provide evidence of where (relative to a Saturn centered reference frame) this feature might have formed, prior to subsequent rotation. However models of crack formation have neglected the role of a physical libration in the calculation of tidal stress. Thus, if this tiger stripe did form at its current location, the modeling of its formation, assuming a physical libration, may provide further evidence for Enceladus' libration state.

\subsection{Global Tidal Dissipation}

A physical libration was first invoked in order to provide greater amounts of heat to the system by allowing Enceladus to tidally dissipate more energy (Wisdom, 2004). The tidal heating is given by

$$
\frac{\mathrm{d} E}{\mathrm{~d} t}=\left[\frac{9}{2} e^{2}+\frac{3}{2}(2 e+F)^{2}+\frac{3}{8} S^{2}+\frac{3}{2}(\sin \varepsilon)^{2}\right] \frac{k}{Q} \frac{G M^{2} n R^{5}}{a^{6}}
$$

where $S$ is the amplitude of the secondary forced libration, assuming that Enceladus is near the 4:1 secondary spin-orbit resonance (Peale and Cassen, 1978; Wisdom, 2004). Wisdom (2004) assumed that the physical libration, F, is out of phase with the optical libration. Thus a more general expression of 
tidal heating (sans our assumptions) would be

$$
\frac{\mathrm{d} E}{\mathrm{~d} t}=\left[\frac{9}{2} e^{2}+\frac{3}{2} L^{2}+\frac{3}{8} S^{2}+\frac{3}{2}(\sin \varepsilon)^{2}\right] \frac{k}{Q} \frac{G M^{2} n R^{5}}{a^{6}}
$$

where $L=\sqrt{4 e^{2}+F^{2}-4 e F \cos (\psi)}$, which is the amplitude of the oscillation in the longitude of the tidal bulge described by $-2 e \sin (n t)+F \sin (n t+\psi)$. When $\psi=\pi, L=2 e+F$ which is the value assumed by Wisdom (2004). If we neglect possible secondary librations $(S=0)$ and assume zero obliquity $(\varepsilon=0)$, then the factor by which tidal dissipation is modified can be expressed as

$$
f_{H}=\frac{3 e^{2}+L^{2}}{7 e^{2}}
$$

The physical libration can enhance or retard tidal dissipation, depending on the value of $L$ (Fig. 8). For cases when $L<2 e$ tidal dissipation is retarded, while enhancement of tidal dissipation occurs when $L>2 e$. If Enceladus' physical libration remains constant in amplitude but slowly migrates in phase over time (a condition that can happen if the libration period is slightly off the orbital period), Enceladus can experience periodic episodes of heating and cooling.

The most enhancement to tidal heating occurs for for the case of a physical libration at Cassini's limit of $1.5^{\circ}$ that is out of phase with the optical libration. In this case the tidal heating is enhanced by $\sim 8.6$ times. Given recent work by Hurford et al. (2007) and Nimmo et al. (2007), which imply at least 8 GW of tidal heating, Enceladus could be dissipating $\sim 70 \mathrm{GW}$, making the $\sim 6 \mathrm{GW}$ seen in the south polar region only a small fraction of the total heat supplied to the system. 


\section{Discussion}

We have shown that a physical libration may have profound effects on geological processes in which tidal stress plays a dominant role. Observations of eruption activity, heat distributions, tectonic displacement and crack shapes (Sections 3.1-3.4, respectively) provide indirect evidence of Enceladus' physical libration. In addition, the Cassini spacecraft provides a platform with several different instruments, especially ISS, that may be able to detect any physical libration.

Observations of the eruption activity on Enceladus provide the best way to detect Enceladus' current libration state (Sec. 3.1). Cassini ISS can directly detect the plumes during high phase imaging. Multiple images, with Enceladus at different points in its orbit, may provide evidence that the eruption rates from Enceladus' south pole varies with orbital position. Cassini UVIS, INMS and MAG observations can also provide handles on the eruption rates and their variability (Hansen et al., 2008; Saur et al., 2008). As those data become available we can expand on the results of Sec 3.1. The changing eruption rates and distribution can be compared to models of how tidal stresses facilitate eruptions, providing more information about the physical libration.

Observations of heat distributions on Enceladus' south polar region can also be used to detect Enceladus' current libration state (Sec. 3.2). Cassini CIRS detects the surface temperatures on Enceladus. Tidal shear heating depends on the diurnal stress field, which is affected by a physical libration. Models of tidal shear heating may be able to match the locations of high temperatures

observed by CIRS. Current models cannot produce enough heat on Baghdad 
but future models, which incorporate a physical libration may better match the CIRS data.

Finally, observations of the geology of the south polar region can provide evidence of Enceladus' physical libration (Sec. 3.3 \& 3.4). Cassini ISS has provided detailed images of the south polar terrain. Modeling the formation of cracks, such as the cycloidal tiger stripe, and strike-slip displacements may provide evidence that a physical libration is needed to best reproduce these feature. However, the additional stress from a physical libration affected these features at the time of their formation, and if the libration state changes with time, it may not be the same for each observation or for that matter may not the same as the current libration state.

All these observational techniques together provide independent methods of characterizing Enceladus' physical libration state. Fig. 9 shows a sketch of these processes and the intersection of their results. Physical libration states that can explain the most observations are more likely to exist. Only by systematically studying each process on its own, can a comprehensive understanding of Enceladus' rotation state can be made.

Although we have focused on Enceladus, physical librations of satellites may be common in the solar system. Recently, work has suggested that winds on Titan may be able to drive a forced libration of Titan's surface (Tokano and Neubauer, 2005), if Titan has a liquid ocean that can decouple its surface from the interior. Moreover, Cassini RADAR observations have been interpreted to suggest that this type of forced libration may exist on Titan (Lorenz et al., 2008). On Europa, the icy crust is almost certainly decoupled from the deeper interior. Recent studies have speculated about the possibility that it also ex- 
hibits a spin-orbit libration as tidal torques from Jupiter may be able to force this ice shell to librate (van Hoolst et al., 2008). Such physical librations on Titan and on Europa, as well as on Enceladus, might also have affects on tidal stress fields, which would also influence geological processes on their surfaces.

\section{Conclusions}

Current efforts to directly detect Enceladus' physical libration limits its amplitude to $\leq 1.5^{\circ}$. However, future observations by Cassini may place further limits on its amplitude and perhaps may even detect the libration. Libration can be detected by observing slight changes in the location of surface features with respect to a) the terminator, b) the limb or c) the spin pole. Detections with respect to the terminator are difficult because topography can obscure the true location of a feature and limb observations suffer from foreshortening. Thus observations of change with respect to the spin pole provide the best chance of observing Enceladus' libration. In order to see the effect of Enceladus' libration a higher resolution control grid, able to detect changes to less than $1^{\circ}$, will be needed.

The effect of a libration, if it exists, may be profound on diurnal tidal stress. A change in the diurnal stress will in turn affect geological processes controlled by these stresses. The location and timing of eruptions will be affected as will the heat generated by tidal shear heating. The formation and modification of cracks on the surface will also be influenced by the stress produced by a physical libration. The ramifications of the diurnal stress on geological processes are more easily observable and may provide the best and only way to indirectly detect Enceladus' librational state. Observations of each process 
can provide its own unique constraint on the libration amplitude and phase. Individually they may not provide a conclusive detection of the libration state. However a much more robust case for physical libration would result if a single model could be constructed to simultaneously account for several geological processes.

\section{References}

Greenberg, R. 2005. Europa - The Ocean Moon: Search for an Alien Biosphere. Published by Springer-Verlag (Springer-Praxis Books in Geophysical Sciences series), Berlin.

Greenberg, R., P. Geissler, G. Hoppa, B. R. Tufts, D. D. Durda, R. Pappalardo, J. W. Head, R. Greeley, R. Sullivan, and M. H. Carr 1998. Tectonic Processes on Europa: Tidal Stresses, Mechanical Response, and Visible Features. Icarus $135,64-78$.

Hansen, C. J., L. Esposito, J. Colwell, A. Hendrix, B. Meinke, and I. Stewart 2008. New Occultation Observation of Enceladus' Plume. In Lunar and Planetary Institute Conference Abstracts, Volume 39 of Lunar and Planetary Inst. Technical Report, pp. 2014.

Hoppa, G., B. R. Tufts, R. Greenberg, and P. Geissler 1999a. Strike-Slip Faults on Europa: Global Shear Patterns Driven by Tidal Stress. Icarus 141, $287-$ 298.

Hoppa, G. V., B. Randall Tufts, R. Greenberg, T. A. Hurford, D. P. O’Brien, and P. E. Geissler 2001. Europa's Rate of Rotation Derived from the Tectonic Sequence in the Astypalaea Region. Icarus 153, 208-213.

Hoppa, G. V., B. R. Tufts, R. Greenberg, and P. E. Geissler 1999b. Formation of cycloidal features on Europa. Science 285, 1899-1902. 
Hurford, T. A., R. Greenberg, and G. V. Hoppa 2006. South Polar Cycloidal Rift on Enceladus. In AAS/Division for Planetary Sciences Meeting Abstracts, pp. \#18.04.

Hurford, T. A., P. Helfenstein, R. Greenberg, and G. V. Hoppa 2007. A Cycloid-like Rift Near Enceladus' South Pole: Europa-style Production by Tidal Stress. In Lunar and Planetary Institute Conference Abstracts, Volume 38 of Lunar and Planetary Institute Conference Abstracts, pp. 1844.

Hurford, T. A., P. Helfenstein, G. V. Hoppa, R. Greenberg, and B. Bills 2007. Eruptions arising from tidally controlled periodic openings of rifts on Enceladus. Nature 447, 292-294.

Leith, A. C., and W. B. McKinnon 1996. Is There Evidence for Polar Wander on Europa? Icarus 120, 387-398.

Lorenz, R. D., B. W. Stiles, R. L. Kirk, M. D. Allison, P. Persi del Marmo, L. Iess, J. I. Lunine, S. J. Ostro, and S. Hensley 2008. Titan's Rotation Reveals an Internal Ocean and Changing Zonal Winds. Science 319, 1649.

Melosh, H. J. 1977. Global tectonics of a despun planet. Icarus 31, 221-243.

Newton, I. 1686. Philosophiae naturalis principia mathematica. Lib. III, Prop. $17 \& 38$. London.

Nimmo, F., and E. Gaidos 2002. Strike-slip motion and double ridge formation on Europa. Journal of Geophysical Research (Planets) 107, 5.

Nimmo, F., J. R. Spencer, R. T. Pappalardo, and M. E. Mullen 2007. Shear heating as the origin of the plumes and heat flux on Enceladus. Nature 447, 289-291.

Peale, S. J., and P. Cassen 1978. Contribution of tidal dissipation to lunar thermal history. Icarus 36, 245-269.

Porco, C. C., P. Helfenstein, P. C. Thomas, A. P. Ingersoll, J. Wisdom, R. West, G. Neukum, T. Denk, R. Wagner, T. Roatsch, S. Kieffer, E. Turtle, 
A. McEwen, T. V. Johnson, J. Rathbun, J. Veverka, D. Wilson, J. Perry, J. Spitale, A. Brahic, J. A. Burns, A. D. DelGenio, L. Dones, C. D. Murray, and S. Squyres 2006. Cassini Observes the Active South Pole of Enceladus. Science 311, 1393-1401.

Prockter, L. M., F. Nimmo, and R. T. Pappalardo 2005. A shear heating origin for ridges on Triton. Geophys. Res. Letters 32, L14202.

Saur, J., N. Schilling, N. Fritz, F. Darrell, S. Simon, M. Dougherty, and C. Russell 2008. Evidence for temporal variability of Enceladus' gas jets: Modeling of Cassini observations. GRL in review 000, 000.

Schubert, G., T. Spohn, and R. T. Reynolds 1986. Thermal histories, compositions and internal structures of the moons of the solar system, pp. 224-292. IAU Colloq. 77: Some Background about Satellites.

Spencer, J. R., J. C. Pearl, M. Segura, F. M. Flasar, A. Mamoutkine, P. Romani, B. J. Buratti, A. R. Hendrix, L. J. Spilker, and R. M. C. Lopes 2006. Cassini Encounters Enceladus: Background and the Discovery of a South Polar Hot Spot. Science 311, 1401-1405.

Spitale, J. N., and C. C. Porco 2007. Association of the jets of Enceladus with the warmest regions on its south-polar fractures. Nature 449, 695-697.

Thomas, P. C., J. A. Burns, P. Helfenstein, S. Squyres, J. Veverka, C. Porco, E. P. Turtle, A. McEwen, T. Denk, B. Giese, T. Roatsch, T. V. Johnson, and R. A. Jacobson 2007. Shapes of the saturnian icy satellites and their significance. Icarus 190, 573-584.

Tokano, T., and F. M. Neubauer 2005. Wind-induced seasonal angular momentum exchange at Titan's surface and its influence on Titan's length-of-day. GRL 32, 24203.

van Hoolst, T., N. Rambaux, Ö. Karatekin, V. Dehant, and A. Rivoldini 2008. The librations, shape, and icy shell of Europa. Icarus 195, 386-399. 
Wisdom, J. 2004. Spin-Orbit Secondary Resonance Dynamics of Enceladus. The Astronomical Journal 128, 484-491.

Yoder, C. F. 1979. How tidal heating in Io drives the Galilean orbital resonance locks. Nature 279, 767-770. 


\section{Appendix A: Diurnal Tidal Stress on a Librating Body}

Variation in the tidal figure of a moon as it orbits produces stress on its surface on a diurnal timescale. The diurnal stress produced by variations driven by orbital eccentricity, obliquity and physical librations, $\sigma^{D}$, is the difference between the stress produced by the tidal figure at any point in its orbit, $\sigma(t)$, and the stress produced by its average tidal deformation, $\sigma$,

$$
\sigma^{D}=\sigma(t)-\sigma=\left|\begin{array}{ll}
\sigma(t)_{x x}-\sigma_{x x} & \sigma(t)_{x y}-\sigma_{x y} \\
\sigma(t)_{y x}-\sigma_{y x} & \sigma(t)_{y y}-\sigma_{y y}
\end{array}\right| .
$$

The stress tensors $\sigma(t)$ and $\sigma$ must be written such that their components describe the tidal stress in a similar reference frame. Stress in the $x x$ direction is aligned north-south while in the $y y$ direction east-west.

The stresses at any latitude $\delta$ and longitude $\lambda$, produced by the thin shell approximation of stress for the average tidal figure $\sigma$, are given by Eq. 1 and Eq. 2 with $\theta=\cos ^{-1}[\cos \delta \cos \lambda]$. The components of the stress tensor $\sigma$ are

$$
\begin{aligned}
\sigma_{x x} & =\frac{1}{2}\left(\sigma_{\phi \phi}+\sigma_{\theta \theta}\right)+\frac{1}{2}\left(\sigma_{\phi \phi}-\sigma_{\theta \theta}\right) \cos 2 \beta, \\
\sigma_{y y} & =\frac{1}{2}\left(\sigma_{\phi \phi}+\sigma_{\theta \theta}\right)-\frac{1}{2}\left(\sigma_{\phi \phi}-\sigma_{\theta \theta}\right) \cos 2 \beta, \text { and } \\
\sigma_{x y} & =\sigma_{y x}=-\frac{1}{2}\left(\sigma_{\phi \phi}-\sigma_{\theta \theta}\right) \sin 2 \beta
\end{aligned}
$$

where the angle $\beta$ describes the tilt of the principal stress axis with respect to the common reference frame and has the value

$$
\beta=\cos ^{-1}\left[-\frac{\sin \delta \cos \theta}{\cos \delta \sin \theta}\right]
$$

The stresses produced the tidal figure at any point along the orbit $\sigma(t)$, are 
given by modified forms of Eq. 1 and Eq. 2, which are valid when $e$ is small,

$$
\sigma(t)_{\theta \theta}=\frac{3 M \mu h_{2}}{8 \pi \rho_{a v} a^{3}}\left(\frac{1+\nu}{5+\nu}\right)(5+3 \cos 2 \theta(t))(1+3 e \cos (n t))
$$

and

$$
\sigma(t)_{\phi \phi}=-\frac{3 M \mu h_{2}}{8 \pi \rho_{a v} a^{3}}\left(\frac{1+\nu}{5+\nu}\right)(1-9 \cos 2 \theta(t))(1+3 e \cos (n t))
$$

where the tidal height and bulge location vary because of the orbital eccentricity $e$ based on the angular position in the orbit defined by $n t$, such that $n$ is the mean motion and $t$ time in seconds after pericenter passage. Moreover, the angular distance between any point on the surface $(\delta, \lambda)$ and the instantaneous location of the tidal bulge $\theta(t)$ is time dependent and described by

$$
\begin{aligned}
\theta(t)=\cos ^{-1} & {[\sin \delta \sin (\varepsilon \sin (n t-\omega))} \\
& +\cos \delta \cos (\varepsilon \sin (n t-\omega)) \\
& \cos (\lambda+2 e \sin (n t)-F \sin (n t+\psi))]
\end{aligned}
$$

where $\varepsilon$ is the obliquity, $\omega$ is the angle between the direction of the ascending node and the direction of pericenter and $F$ is the amplitude of the physical libration with phase $\psi$. Here we focus on the physical libration that is driven at the orbital frequency, free librations may occur at frequencies other than the orbital frequency and those librations can be added to $\theta(t)$ by adding terms similar to $-F \sin (n t+\psi)$ which describe the free librations.

The components of the stress tensor $\sigma(t)$ are

$$
\begin{aligned}
\sigma_{x x}(t) & =\frac{1}{2}\left(\sigma_{\phi \phi}(t)+\sigma_{\theta \theta}(t)\right)+\frac{1}{2}\left(\sigma_{\phi \phi}(t)-\sigma_{\theta \theta}(t)\right) \cos 2 \beta(t), \\
\sigma_{y y}(t) & =\frac{1}{2}\left(\sigma_{\phi \phi}(t)+\sigma_{\theta \theta}(t)\right)-\frac{1}{2}\left(\sigma_{\phi \phi}(t)-\sigma_{\theta \theta}(t)\right) \cos 2 \beta(t), \text { and } \\
\sigma_{x y}(t) & =\sigma_{y x}(t)=-\frac{1}{2}\left(\sigma_{\phi \phi}(t)-\sigma_{\theta \theta}(t)\right) \sin 2 \beta(t)
\end{aligned}
$$


where the angle $\beta(t)$ describes the tilt of the instantaneous principal stress axis with respect to the common reference frame and is defined as

$$
\beta(t)=\cos ^{-1}\left[\frac{\sin (\varepsilon \sin (n t-\omega))-\sin \delta \cos \theta(t)}{\cos \delta \sin \theta(t)}\right] .
$$

With the formulation of $\sigma$ and $\sigma(t)$ in similar reference frames, the diurnal stress, $\sigma^{D}$, can now be evaluated. 


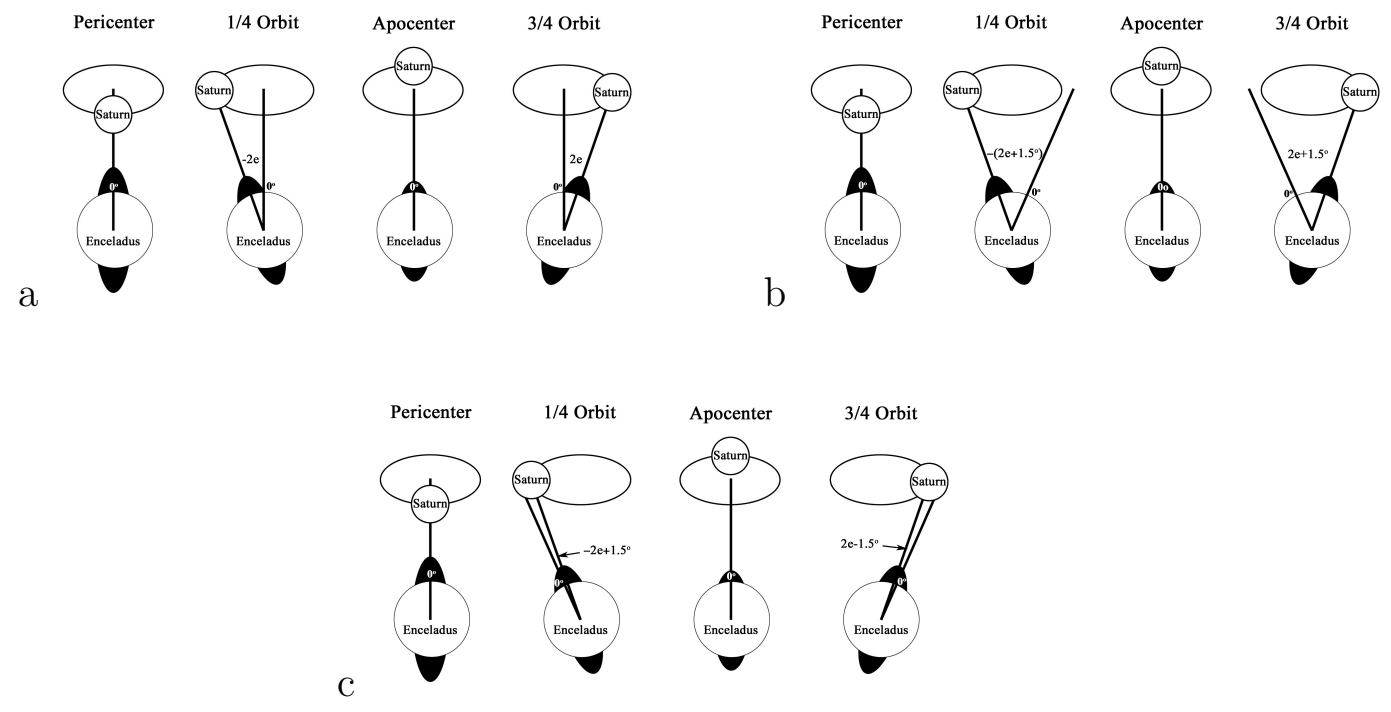

Fig. 1. Sources of stress on Enceladus. The tidal bulge raised on Enceladus by Saturn (in black) changes both its position and its magnitude as the satellite orbits the planet. These effects are shown in (a) in a reference frame centered on Enceladus that rotates at the satellite's mean orbital rate $n$. In addition, a hypothetical physical libration of $1.5^{\circ}$, either out of phase (b) or in phase (c) with the motion of Saturn, would cause a periodic nodding of the satellite relative to a uniform spin state. Straight lines from Enceladus indicate how the direction to Saturn and the zero point of a longitude grid fixed to Enceladus change with time. While the position of the tidal bulge relative to landmarks on Enceladus depends on both eccentricity and physical libration, the magnitude of the tidal bulge is affected only by the first process. In the special case that Enceladus exhibits an in-phase physical libration with an amplitude of $2 e$, the longitude of the tidal bulge is fixed to $0^{\circ}$ similar to panel (c). 
Stress Field from Eccentricity at $1 / 4$ of an orbit

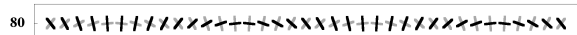

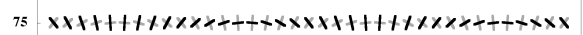

$70-x \times t++++1 \times x \times x++++x \times x x+t+++x \times x \times x++++x \times x$

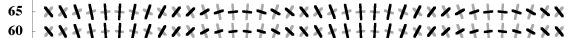

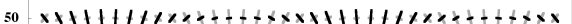

$40 \times x|||| 11 x+4+t+6 x+1|| l 11 x+4+t+6 x$

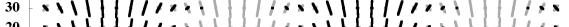

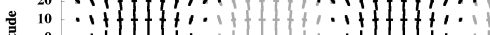

焉 -10

, 1 , $1 \cdots$, itt

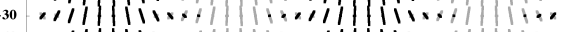

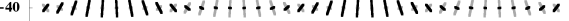

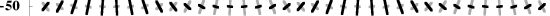

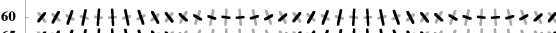

$-70 \times x \times 1+t+7 \times x \times x+4++x \times x \times x+t+7+x \times x \times x+++x \times x$

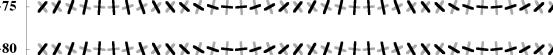

$\begin{array}{lllllllllllll}360 & 330 & 300 & 270 & 240 & 210 & 180 & 150 & 120 & 90 & 60 & 30 & 0\end{array}$

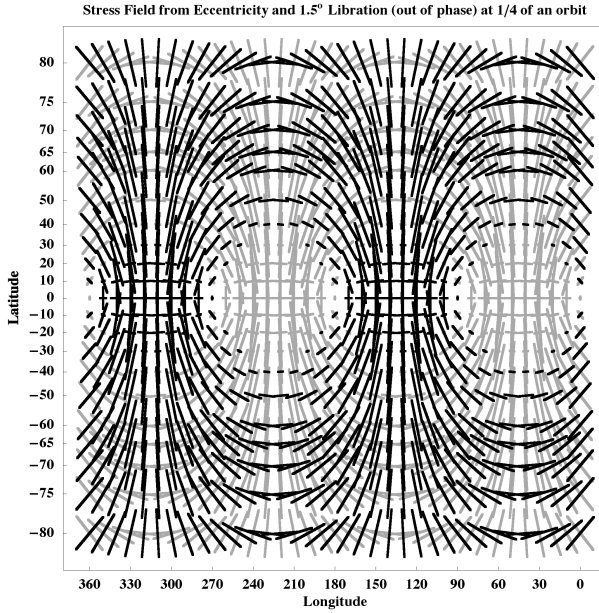

$\mathrm{b}$

Fig. 2. 


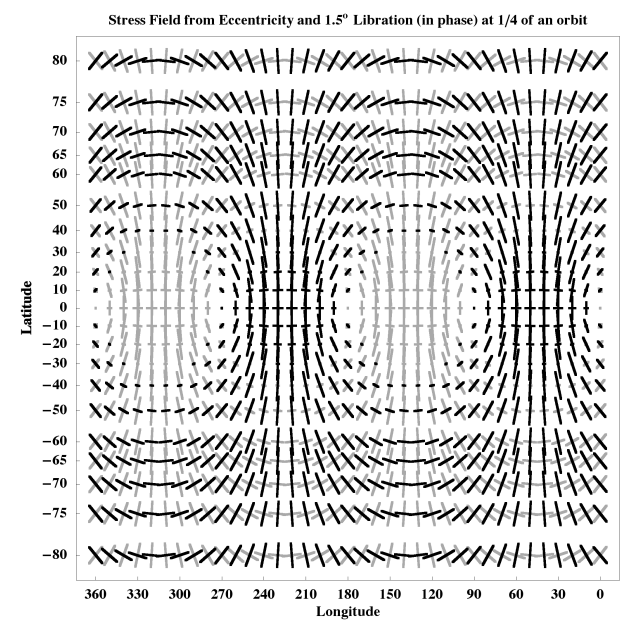

$\mathrm{C}$

Fig. 2. Here we illustrate the tidal stress on Enceladus 1/4 of an orbit past pericenter ( $n t=\pi / 2)$ for the three cases of Fig. 1. Dark lines represent tension, while lighter lines show compression. As in Fig. 1, (a) corresponds to the optical libration of the tide due solely to Enceladus' orbital eccentricity. To this, we add in (b) an out-of-phase physical libration with amplitude of $1.5^{\circ}$ and in (c) an in-phase $1.5^{\circ}$ physical libration. With an in-phase physical libration of amplitude $2 e$, the positions of the tidal bulges are fixed on Enceladus and stresses arise only from their amplitude variations. The stress fields at $(n t=3 \pi / 2)$ can be obtained from the ones shown here by interchanging the meaning of the dark and light lines, so that the former represents compression while the latter shows tension. 


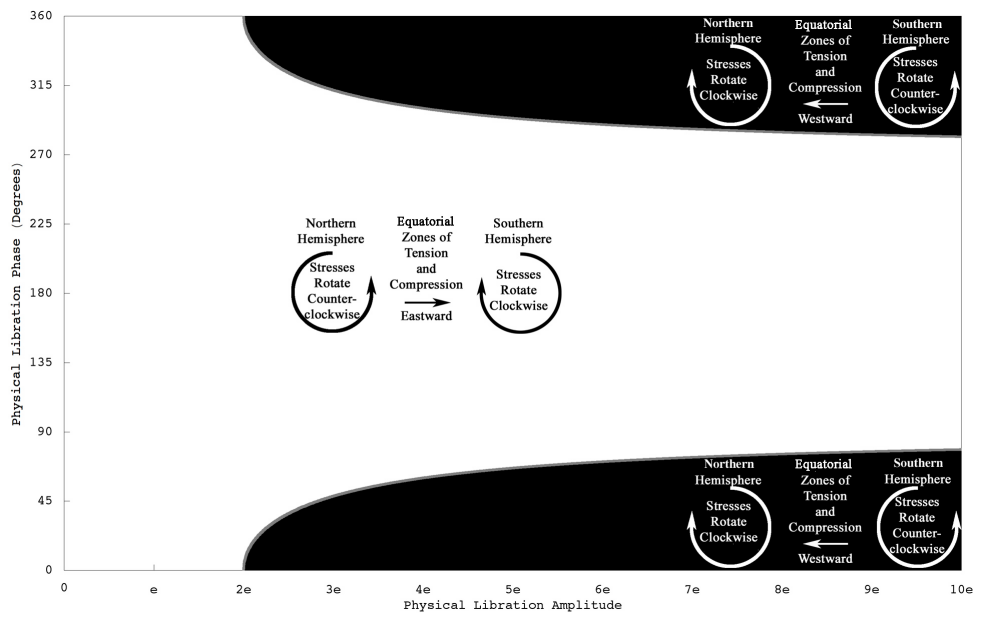

Fig. 3. Here we plot the phase of the physical libration against its amplitude. In the white region, tidal stress the orientation of the principal stresses rotate in a counterclockwise fashion in the northern hemisphere and clockwise in the southern hemisphere while zones of compression and tension move towards the east along the equator. In the black region that pattern is reversed. Finally, along the gray border between the white and black region, the tidal stress field changes its sense of rotation at pericenter and apocenter and the zones of tension and compression oscillate about a fixed longitude. This figure is general and threfore applicable to any librating satellite. 


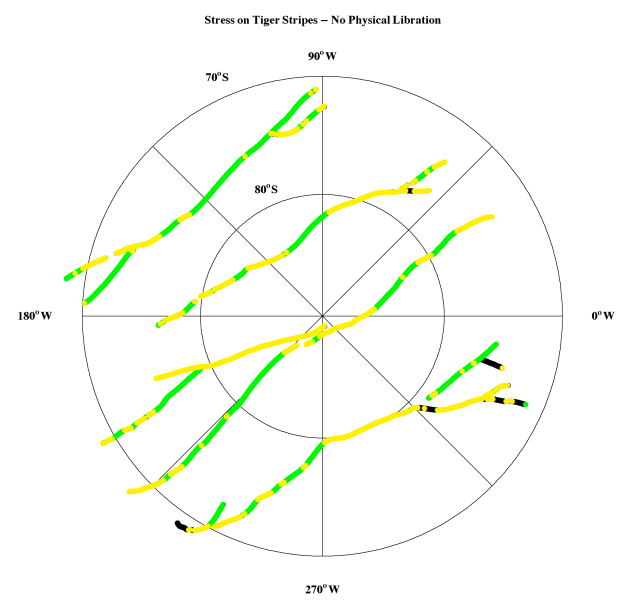

a

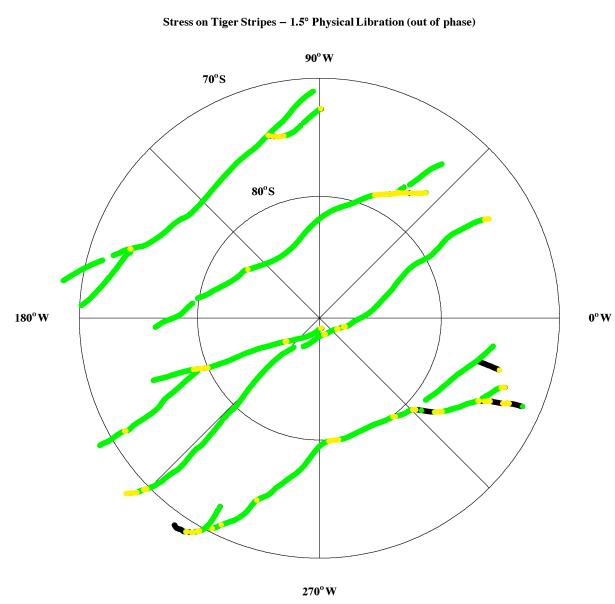

b

Fig. 4. 


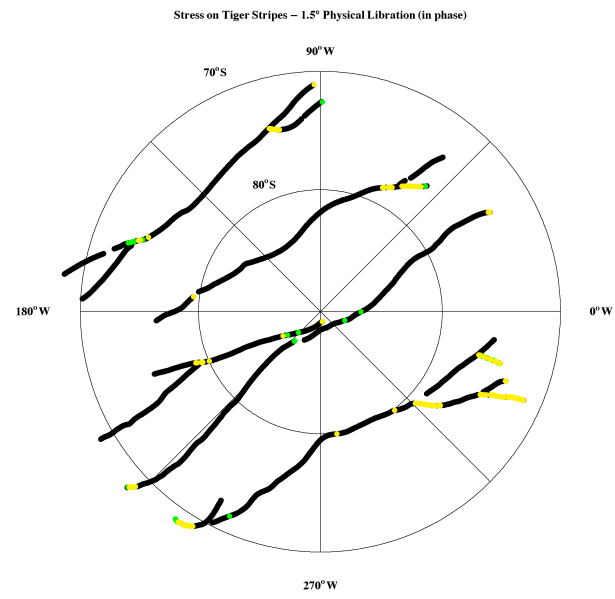

$\mathrm{C}$

Fig. 4. The stress state across the tiger stripe rifts is shown during the period $\pi / 4<n t<\pi / 2$ (second eighth of the orbit) for the cases in Fig. 1a-c. The color indicates the stress state. Black means that portion of the feature was in compression during the entire portion of the orbit while green means that it was in tension. Yellow indicates that the stress switched from compression to tension, opening the rift during this period. 


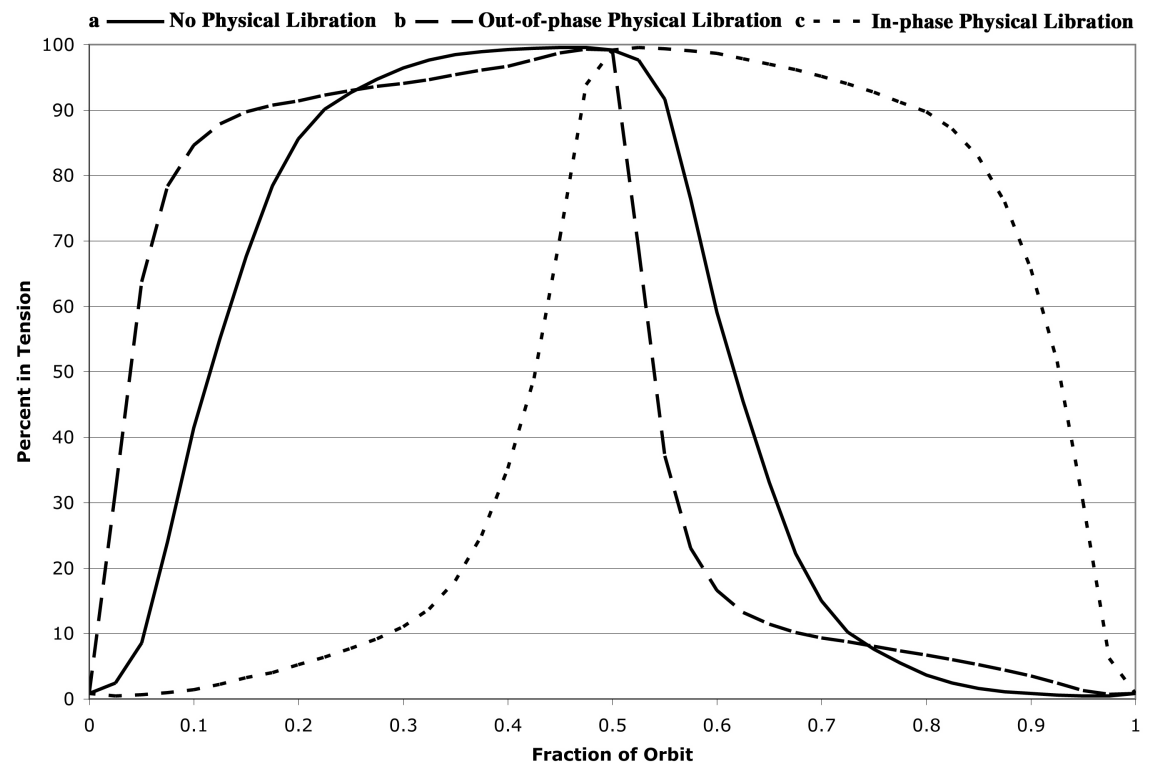

Fig. 5. The fraction of the tiger stripes in tension throughout the orbit for the cases in Fig. 1a-c. Eruption rates may be linked to the amount of cracks in tension, creating variation in the plumes throughout the orbit. Different types of physical libration change the timing in when cracks experience tension and may also affect eruption rates throughout the orbit. 


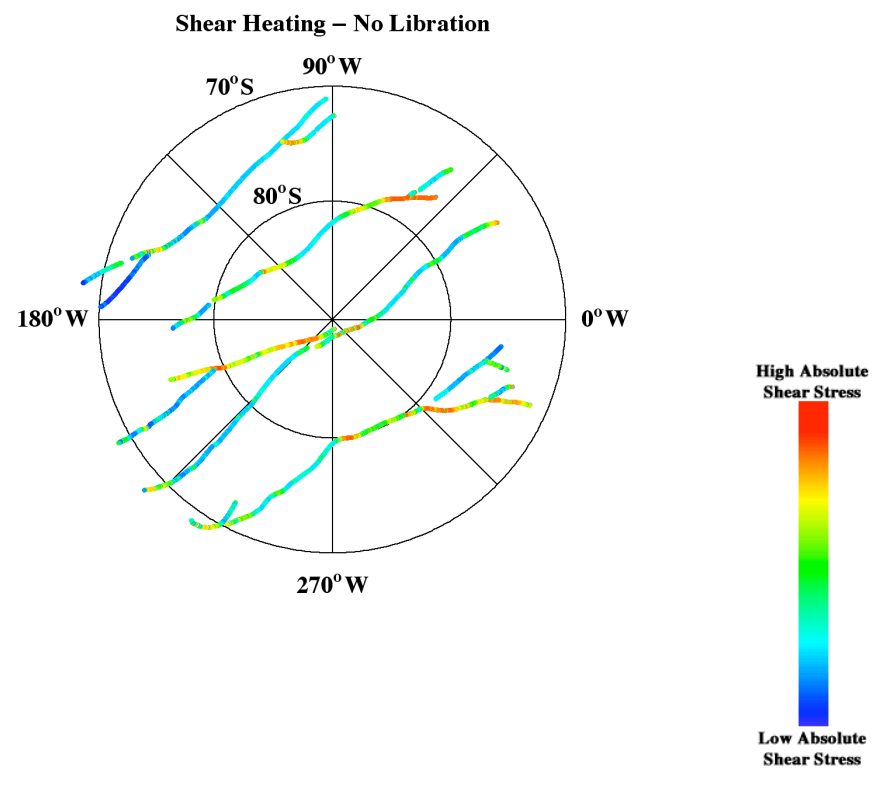

Fig. 6. 

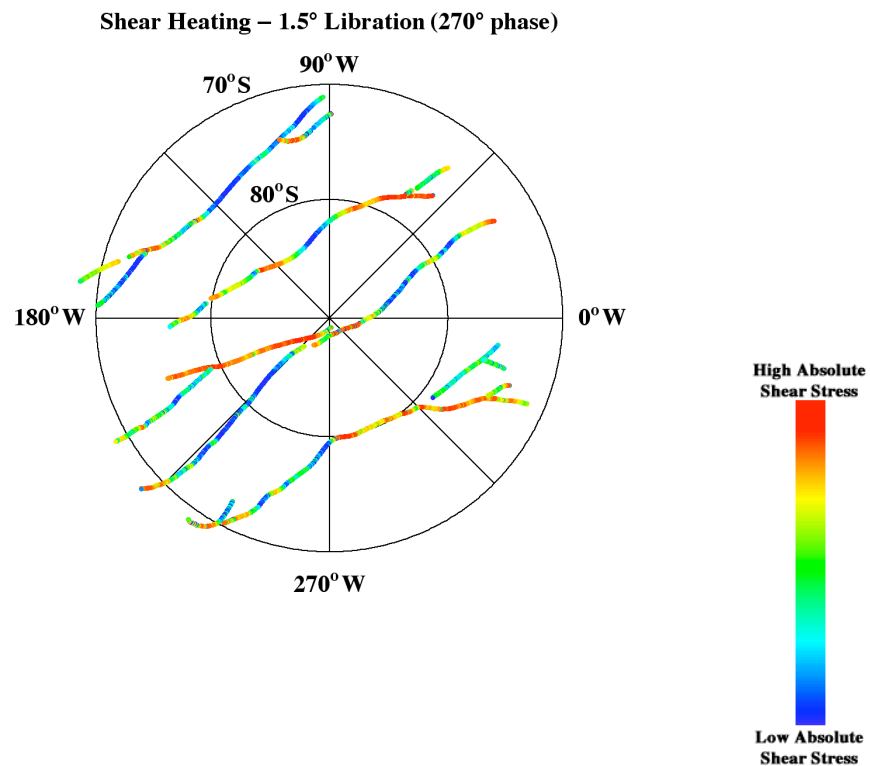

Fig. 6. Absolute tidal shear has been calculated along the tiger stripes in the south polar region for the case when Enceladus has (a) no physical libration, only an optical libration and (b) a libration of amplitude $1.5^{\circ}$ that is out of phase with the optical libration by $270^{\circ}$. In the first case, our results match those of Nimmo et al. (2007). In the second case, areas of greater shearing shown in (a), and thus presumably the locations of hot spots, remain. However, more shearing is seen near the south pole along Baghdad, predicting more tidal shear heating in that region, which matches some aspects of CIRS observations better. Note: Each plot has a different dynamic range between hot and cold colors. 


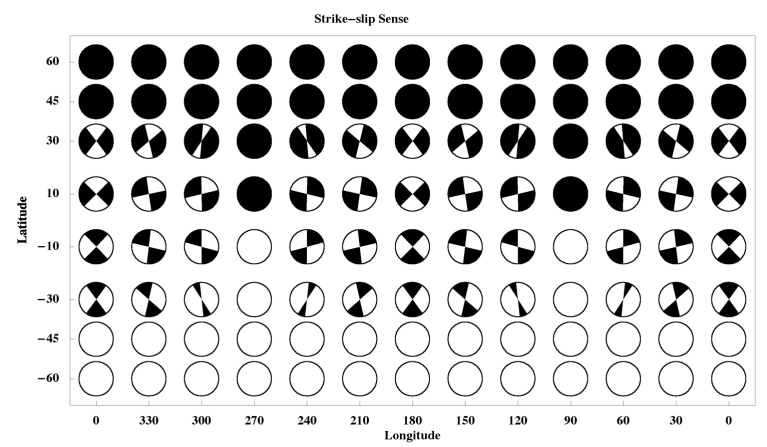

Fig. 7. This plot, used in conjunction with Fig. 3, shows the effect of libration on strike-slip displacement. Here right-lateral displacement is shown as white (left-lateral displacement as black) for values of the physical libration amplitude and phase that define the white region in Fig. 3. For parameters falling in the black region of Fig. 3, the meanings of white and black in this figure are reversed. Along the grey margin between white and black regions in Fig. 3 no strike-slip displacement occurs along any faults of any orientation. 


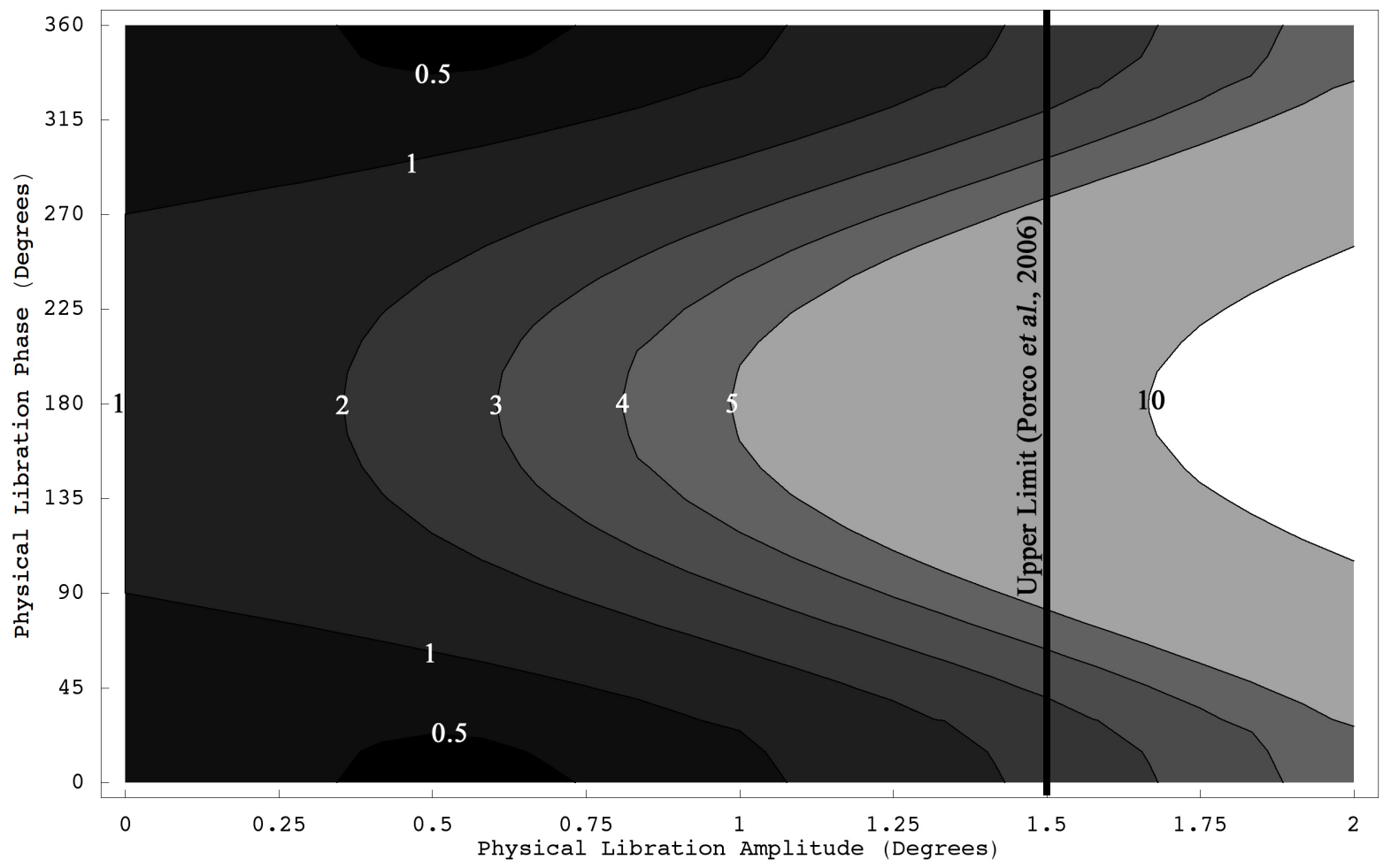

Fig. 8. The factor $f_{H}$ (Eq. 6) by which heating is enhanced or reduced is shown for different values of the physical libration amplitude and its phase relative to the optical libration. In most cases tidal heating is enhanced by the presence of a physical libration; the heating is minimum at $43 \%$ for an in-phase physical libration with amplitude $F=2 e=0.54^{\circ}$. 


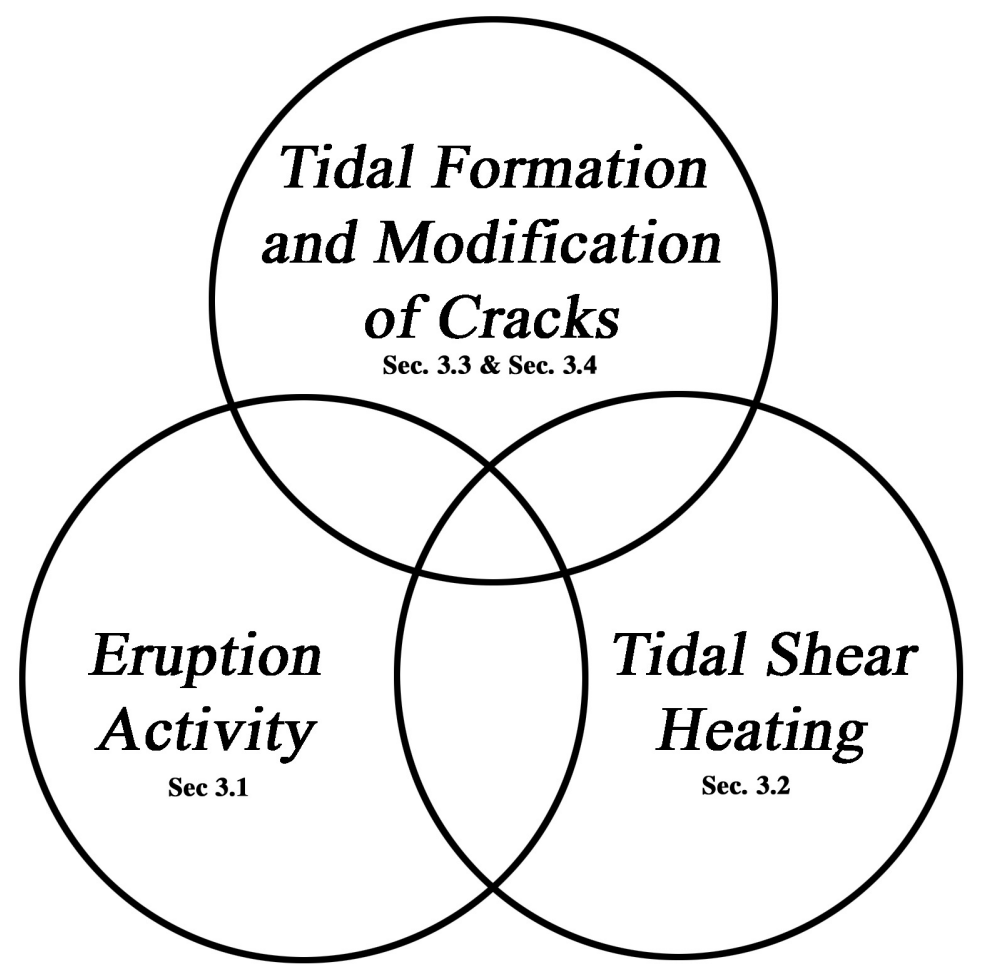

Fig. 9. Possible consequences of the libration of Enceladus. If some or all of these processes are controlled by libration, they could provide powerful constraints on the amplitude and phase of this motion. 